\title{
Development and Applications of Fluorescent Proteins for Correlative Light and Electron Microscopy
}

Maria G. Paez-Segala ${ }^{1}$, Yalin Wang ${ }^{2}$, Nirmala Iyer ${ }^{1}$, Wei-Ping Li $^{1}$, Patricia K. Rivlin ${ }^{1}$ and Loren L. Looger $^{1}$

${ }^{1 .}$ Howard Hughes Medical Institute, Janelia Research Campus, Ashburn, VA.

2. Tsinghua University, Beijing, China.

Recent advances in super-resolution microscopy have pushed the resolution limit of light microscopy closer to that of electron microscopy. However, as they invariably rely on fluorescence, light microscopy techniques only visualize whatever gets labeled. On the other hand, while electron microscopy reveals cellular structures at the highest resolution, it offers no specificity. The information gap between the two imaging modalities can only be bridged by correlative light and electron microscopy (CLEM). Previously we have developed a probe (mEos4) whose fluorescence and photoconversion survive $0.5-1 \% \mathrm{OsO}_{4}$ fixation, allowing super-resolution visualization of organelles and fused proteins in the context of resinembedded ultrastructure in both transmission EM (TEM) and scanning EM (SEM) $[1,2]$.

mEos4 was useful for studying protein localization in good-ultrastructure resin-embedded EM of cultured cells. We seek to optimize reagents and protocols for high-quality CLEM in brains of model organisms (e.g. fly, mouse), to facilitate neuronal reconstruction and localization of synapses, receptors, etc. To that end, we made fly lines expressing the mEos4 variants and prepared them using whole-brain histology techniques adapted to take advantage of fixation-resistant fluorophores. We screened a panel of resins including Epon 12, Quetol, GMA, HM20, in conjunction with chemical fixation or high-pressure freeze/freeze substitution. Promising results were obtained with several combinations on both adult and larval flies (example shown in Fig. 1).

In parallel, we are continuing to optimize the Eos4 line of proteins to improve: fixation resistance of the green state (good in Eos4); fixation resistance of the converted red form (poor); 2-photon photoconversion (poor) and label density in thin structures such as axons and dendrites. Current Eos variants show improved preservation of the converted red form after $\mathrm{OsO}_{4}$ fixation (Fig. 2a) and more than doubling of the contrast ratio (Fig. 2b).

The improved reagents and protocols discussed will enable more CLEM experiments, both on cultured cells and on intact samples.

References:

[1] Paez-Segala et al, Nat Methods (2015).

[2] Kopek et al, Nat Protoc (2017). 


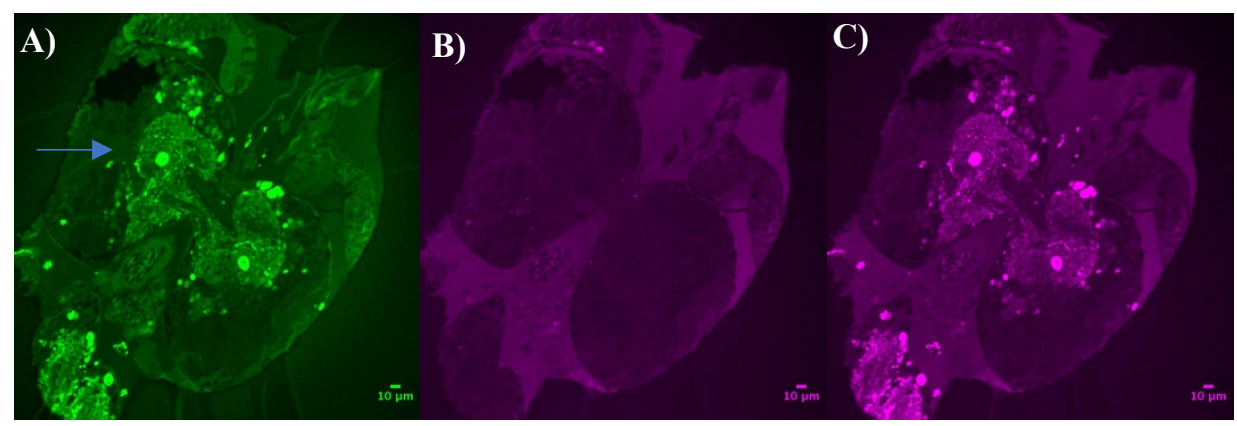

Figure 1. mEos4 expressing in the cytoplasm of neurons in the optic lobe of Drosophila $3^{\text {rd }}$ instar larval $\mathrm{CNS}$. Sample has been treated using $\mathrm{HPF} / \mathrm{FS}$ and $0.5 \% \mathrm{OsO}_{4}$, with $\mathrm{GMA}$ resin infiltration. A-B) green/red fluorescent image before conversion, and $\mathbf{C}$ ) red fluorescent image after conversion with UV light.Blue arrow points optic lobe.

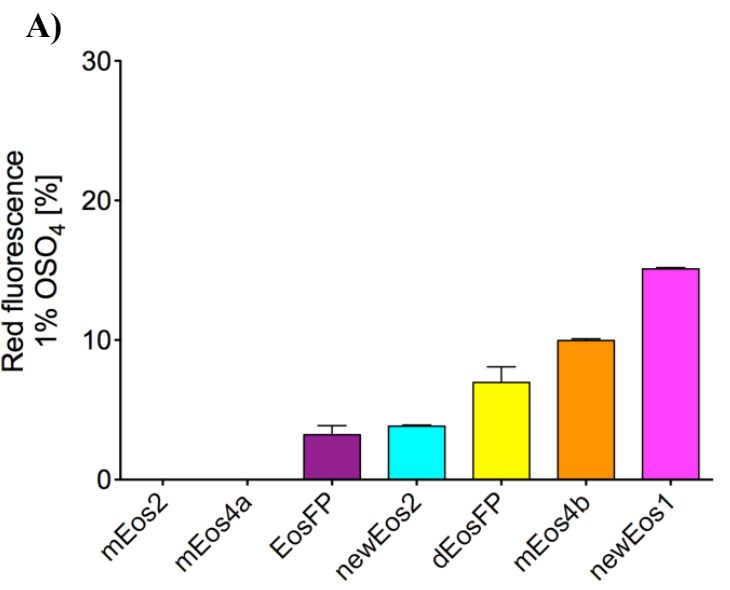

B)

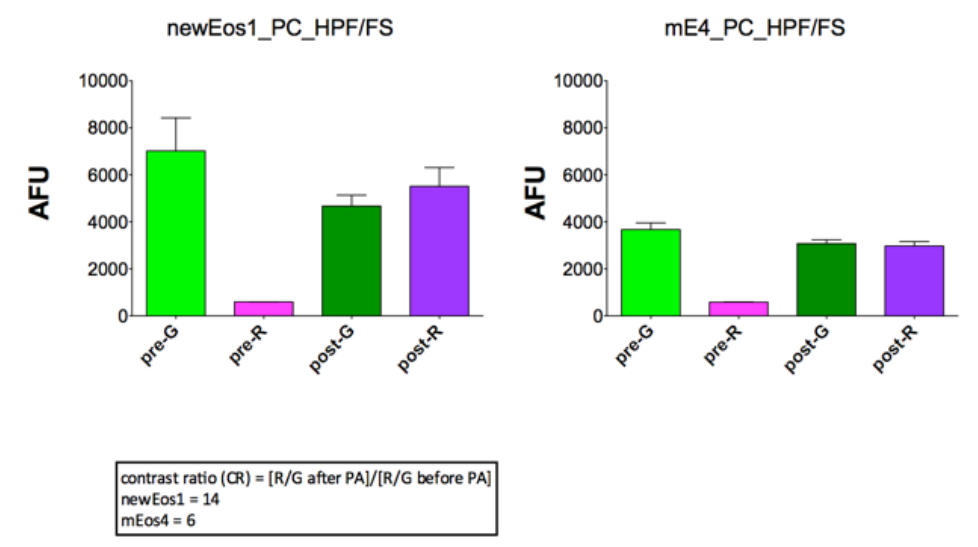

Figure 2. A) Results from a cell-free assay of photoconverted red fluorescence survival after $\mathrm{OsO}_{4}$ incubation (Ex. $550 \mathrm{~nm} / \mathrm{Em} .580 \mathrm{~nm}$; bandwidth $5 \mathrm{~nm} / 5 \mathrm{~nm}$ ). Fluorescence was measured from the bottom after conversion. B) Left graph shows fluorescence survival of a hippocampal rat neuron section expressing tEos 4 in the cytoplasm that has been treated using $\mathrm{HPF} / \mathrm{FS}$ and $0.5 \% \mathrm{OsO}_{4}$, with GMA resin infiltration. Right graph shows the same but for mEos4. Contrast ratio (defined as red/green ratio after photoconversion normalized to red/green ratio before photoconversion) is shown in the box below. $\mathrm{PC}=$ photoconversion; pre- $\mathrm{G}=$ green fluorescence before photoconversion with UV light. Post- $\mathrm{G}=\mathrm{Green}$ fluorescence after photoconversion; HPF-FS = high pressure freezing and freeze substitution. 